\title{
A Comparative Study to Evaluate Periapical Pathology Using Mid Field of View CBCT and Direct Digital Radiography
}

\author{
Deepigaa Manivasagam ${ }^{1}$, Arvind Muthukrishnan ${ }^{2}$ \\ 1,2 Department of Oral Medicine and Radiology, Saveetha Institute of Medical and \\ Technical Sciences (SIMATS), Kuthambakkam, Tamil Nadu, India.
}

\section{ABSTRACT}

\section{BACKGROUND}

Local response of the bone surrounding the apex of the tooth as a result of pulp necrosis or destruction of the periapical tissues caused by significant periodontal disease is known as a periapical inflammatory lesion. Intraoral radiography is the most commonly used technique but has limitations in representing only 2dimensional images. СBCT was created primarily to provide 3-dimensional maxillary skeletal images and a smaller and mid field of view (FOV) have a higher spatial resolution and improved diagnostic potential. The intention of this study was to compare and evaluate the results of limited view CBCT and DDI in the diagnosis of periapical pathology.

\section{METHODS}

In this study, a total of 25 patients who visited the oral medicine department with clinical and or radiographic findings were included. Periapical lesions were assessed using a cone-beam CT periapical index (CBCTPAI) scoring system in both direct digital imaging (DDI) radiographs and CBCT images.

\section{RESULTS}

Periapical lesions were found to be more prevalent in 30 - 39 years (40 \%) with a male predilection (64\%) and maxillary anterior (36\%) more commonly affected. Wilcoxon signed-rank test performed to assess the mean difference between the two imaging modalities revealed a $\mathrm{P}<0.001$ and was statistically significant.

\section{CONCLUSIONS}

This study highlights the role of CBCT in diagnosing periapical lesions which can be missed or misdiagnosed on DDI.

\section{KEY WORDS}

Cone Beam Computed Tomography (CBCT); Direct Digital Imaging (DDI); Periapical Lesions
Corresponding Author: Dr. Arvind Muthukrishnan, Saveetha Institute of Medical and Technical Sciences (SIMATS), Kuthambakkam, Tamil Nadu, India.

E-mail: arvindm@saveetha.com

DOI: $10.14260 /$ jemds $/ 2021 / 742$

How to Cite This Article:

Manivasagam D, Muthukrishnan A. A comparative study to evaluate periapical pathology using mid field of view CBCT and direct digital radiography. J Evolution Med Dent Sci 2021;10(43):3659-3664, DOI: 10.14260/jemds/2021/742

Submission 16-07-2021, Peer Review 24-08-2021, Acceptance 01-09-2021, Published 25-10-2021.

Copyright (C) 2021 Deepigaa Manivasagam et al. This is an open access article distributed under Creative Commons Attribution License [Attribution 4.0 International (CC BY 4.0)] 


\section{BACKGROUND}

The periapical inflammatory lesion is considered to be a local bone response in the area around the apex of the tooth, caused by pulp necrosis or periapical tissue destruction as a result of extensive periodontal disease. ${ }^{1}$ It is always challenging in diagnosing periapical lesions and assessing the size and nature of periapical lesions is essential to determine the treatment and prognosis of the tooth. ${ }^{2}$ The clinician should have adequate information about the extent of the lesion, the morphology of the affected tooth including the number of roots and root canals. ${ }^{3}$ Biopsy is the gold standard in diagnosing and differentiating granulomas from cysts. ${ }^{4}$ In order to diagnose periapical lesions, biopsies cannot be used as routine diagnostic procedures and monitoring their treatment results. ${ }^{5}$ The imaging technique should be capable of detecting the lesion extent, thickness and degree of mineralisation, good resolution and imaging geometry. ${ }^{6}$

Intraoral radiographs are the most commonly used diagnostic tools and are easily available. They work on the principle of compressing a three-dimensional object into a two-dimensional image and the clinician should recreate the image in three dimensions mentally. ${ }^{7}$ Surrounding the structure of interest there is a black ground pattern that creates a complex background making the structure of interest difficult to interpret. ${ }^{8}$ Literature states that medullary bone has a low mineral content thus large resorptive lesions can be undetected and lesions occurring within the cancellous bone have masking making its identification difficult.6,9,10 Eickholz and Haumann reported that underestimation of bone loss by periapical radiographs may range up to 1.41 to $2.58 \mathrm{~mm}$ and they also fail to detect the actual size of the lesion. ${ }^{11}$ Direct digital imaging (DDI) is a great revolution of radiology that works as a result of the image acquisition process and development of network computing systems. ${ }^{12}$ The advantages of DDI, when compared to conventional radiographs, are reduced dose, faster image acquisition, reduced processing errors, comparable resolution and contrast adjustments. ${ }^{13}$

3D imaging was carried out in endodontics using a computed tomography (CT) but they did not reveal adequate diagnostic information, in addition they were expensive, yielded high radiation dose and were not easily available. ${ }^{14}$ Thus in 2000 there was the invention of cone-beam CT (CBCT) which helped in imaging of the dental hard tissues three-dimensionally. ${ }^{15}$ They are capable of producing a higher diagnostic image with a resolution of 2 line pairs / $\mathrm{mm}$, slice thickness of 0.125 to $2.00 \mathrm{~mm}$ with a shorter scanning time of $60 \mathrm{~s} .{ }^{16}$ In comparison to conventional CT the radiation dose is 10 times lesser as they have a single 360 degree scanning projection and isotropic voxels which are equal in all three proportions. ${ }^{17}$ They have been widely used for implant planning, assessment of impacted teeth, temporomandibular joint disorders and endodontics. ${ }^{18}$ Field of view (FOV) can be optimised for the patient depending on the presentation of the disease. ${ }^{19}$ Periapical regions are better visualised with a smaller FOV as they have increased resolution to improve the diagnostic accuracy by detecting periapical pathosis in patients with no specific symptoms, the extent of the lesion in all directions, complex tooth anatomy, calcifications, fractures, external and internal root resorption and bone loss. ${ }^{20}$
The goal of this study was to compare and evaluate the results of limited view CBCT and DDI in the diagnosis of periapical pathology.

\section{METHODS}

From February 2018 to December 2019, the prospective diagnostic study was conducted at a university dental hospital. Both the institutional and the scientific review boards approved the study (SRB). 2 people were involved in the research, a primary researcher and a faculty. Based on the prevalence of periapical lesions reported in our department sample size was calculated to the power of 80 using G power analysis software.

1. Symptomatic teeth (pain, discomfort) and / or

2. Relevant clinical findings (grossly decayed, mobility, tender on percussion, intra oral / extraoral swelling and fistula) and / or

3. Incidental radiographic finding of periapical lesion.

\section{Exclusion Criteria}

1. Pregnant women

2. Deciduous teeth

3. Children below 12 years of age

A paralleling technique was used to obtain DDI radiographs using a Kodak RVG 5200 and a size 2 intraoral digital sensor was used and the machine operated at $70 \mathrm{kVp}$, $8 \mathrm{~mA}$ and 0.32s. The X-ray tube head was held at right angles to both the tooth and the sensor, with the sensor parallel to the long axis of the tooth to be inspected. The image was analysed by adjusting the contrast, brightness, edge enhancement, pseudo colours and zoom controls for better visualisation. CBCT examinations were performed using Sirona Orthophos XG 3D digital imaging system. The occlusal plane was parallel to the scan plane, and the midsagittal plane was perpendicular to the floor. Images were obtained at 85 $\mathrm{kVp}, 10 \mathrm{~mA}, 15 \mathrm{~s}$ with a voxel size of $100 \mu \mathrm{m}$ and the FOV was $15 \mathrm{X} 12 \mathrm{~cm}$ with a $200 \mu \mathrm{m}$. The images were examined using Sirona 3D software. The images were sliced into three dimensions and were analysed in all three planes.

A cone-beam CT periapical index (CBCTPAI) is a scoring system for radiographic assessment of periapical lesions. Score 0 is given for intact bone structures, score 1 for periapical radiolucency $>0.5-1 \mathrm{~mm}$, score 2 for periapical radiolucency $>1-2 \mathrm{~mm}$, score 3 - periapical radiolucency $>2$ - $4 \mathrm{~mm}$, score 4 - periapical radiolucency $>4-8 \mathrm{~mm}$, score 5 periapical radiolucency $>8 \mathrm{~mm} .{ }^{21}$

A total of 25 patients with clinical and / or radiographic abnormalities were included in this investigation. Internal validity was maintained as a result of follow-up on defined diagnostic criteria, which could be repeated in other centres while maintaining external validity.

\section{Statistical Analysis}

The data for the study was first compiled on an excel sheet before being transferred to an SPSS file. In our study, IBM SPSS 20 was used. Gender and tooth affect the qualitative variables used in the study. Quantitative variables include the 
age and CBCTPAI index. The chi-square test was used to determine the correlation and association for age, gender, and tooth affected. A Wilcoxon signed-rank test was used to compare the scores obtained from the CBCTPAI index between the two imaging modalities.

\section{RESULTS}

25 patients with DDI and CBCT images have been included in our study. The study population's most common age group and gender distribution was 30 - 39 years ( $40 \%$ ) followed by 20 - 29 years (28\%); 40 to 49 years $(24 \%)$ and 50 to 59 years (8\%) with a male predilection of $64 \%$ followed by females (36\%). [Graph 1]
Periapical lesions were reported maximum in maxillary anteriors (36 \%) followed by maxillary premolars (24\%); mandibular anteriors (16 \%); maxillary molars (8 \%); mandibular premolars (8\%) and molars (8\%). [Graph 2]

A chi-square test was used to determine the relationship and association of age and gender with tooth affected which revealed that the maxillary anteriors were more affected in 20 - 29 years; maxillary premolars and mandibular anteriors in 30 to 39 years and $P>0.05$. Maxillary anterior and molars were reported more in males while mandibular anteriors in females; maxillary and mandibular premolars were seen equally in both genders [Graph 3 and 4].

Mean and standard deviation of DDI and CBCT were 1.40 \pm 1.118 and $2.52 \pm 1.388$ respectively. Wilcoxon signed-rank test performed to compare the CBCT PAI scores between the DDI and CBCT imaging modality revealed $\mathrm{P}<0.001$ and thus was statistically significant.[Table 1]
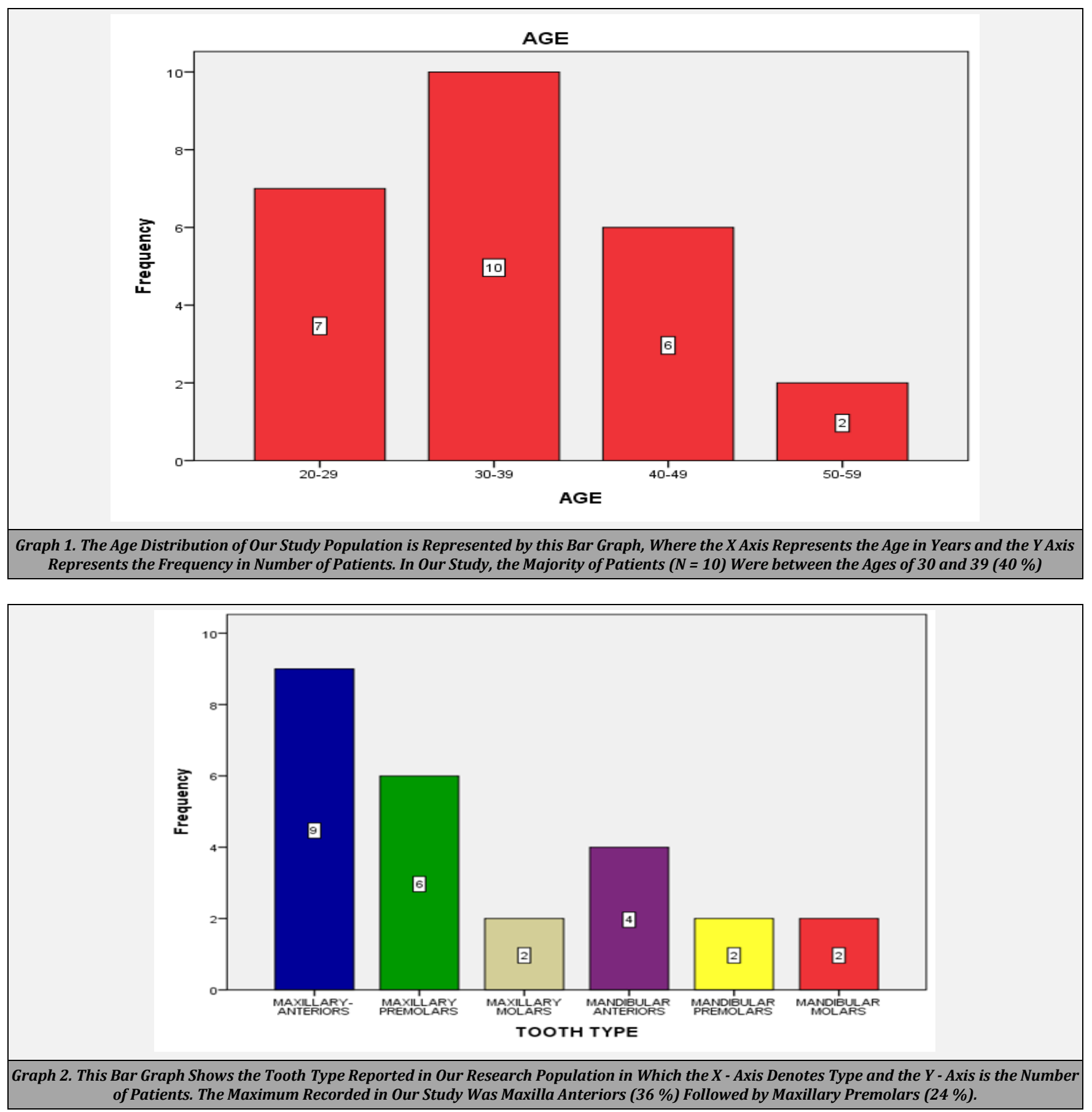


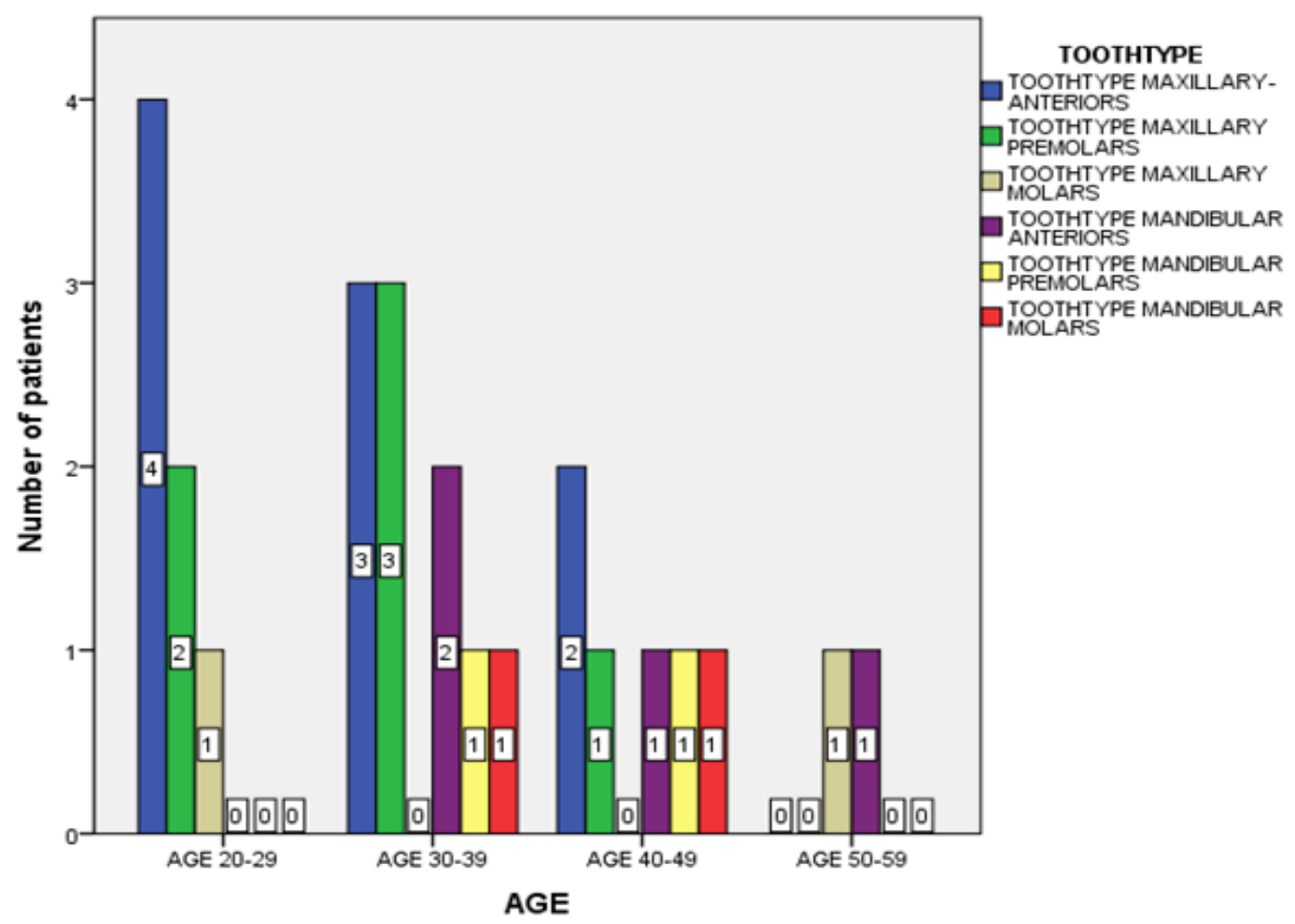

Graph 3. This Bar Graph Denotes the Association of Age and Tooth Affected, the X Axis Denotes the Age in Years and Y Axis Denotes the Patients in Number. Maxillary Anteriors Were More Affected in 20 - 29 Years; Maxillary Premolars and Mandibular Anteriors between the Ages of 30 to 39 . Chi Square Pearson $=13,896(P>0.05)$ (Chi - Square), Which is Not Significant Statistically $(P>0.05)$.

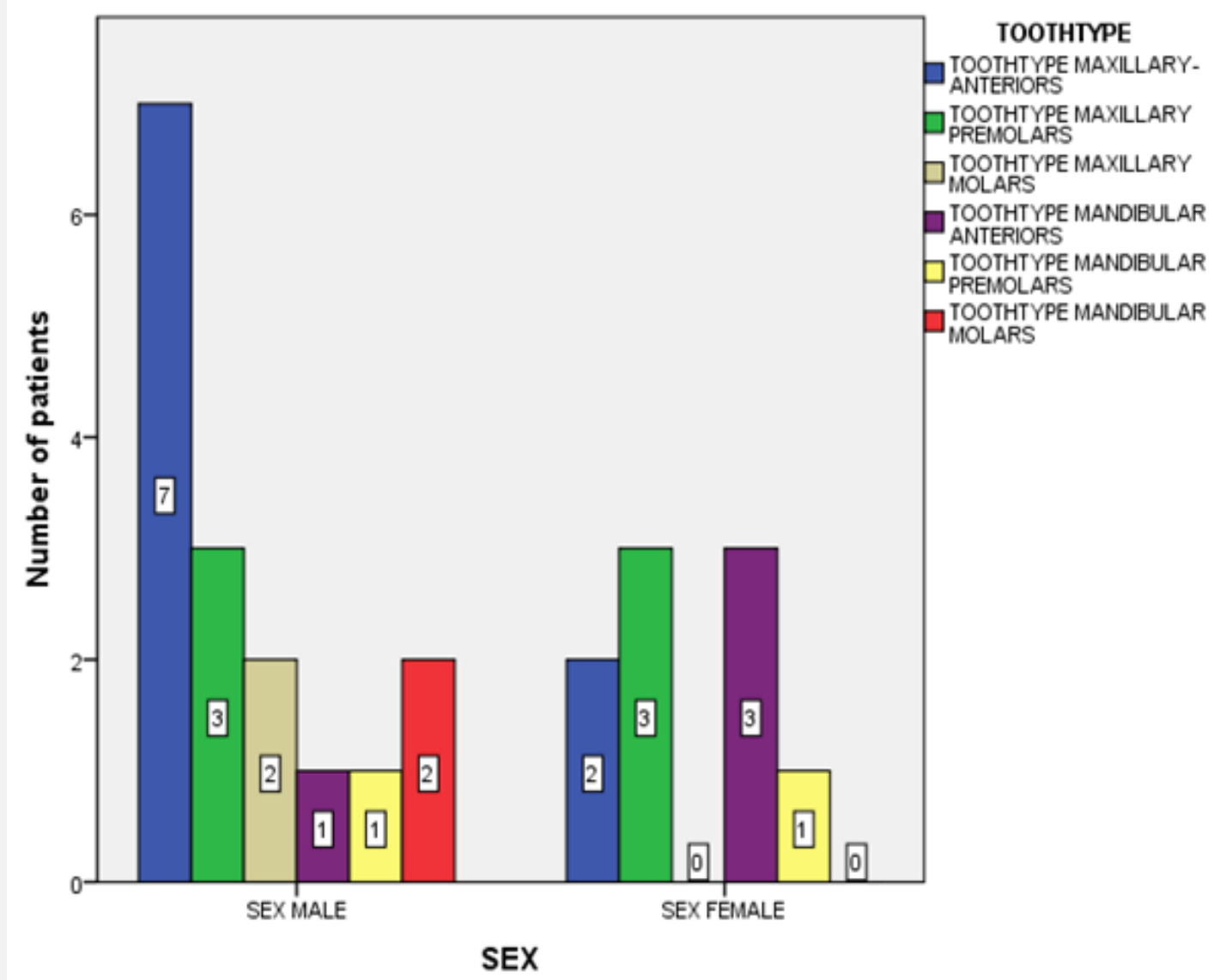

Graph 4. The X Axis Represents Gender and the Y Axis Represents the Number of Patients in this Bar Graph. Males Had More Maxillary Anteriors and Molars, While Females Had More Mandibular Anteriors; Both Genders Had Equal Number of Maxillary and Mandibular Premolars. The Pearson Chi Square $=6.313 P=0.277(P>0.05)$ Was Statistically Insignificant. 


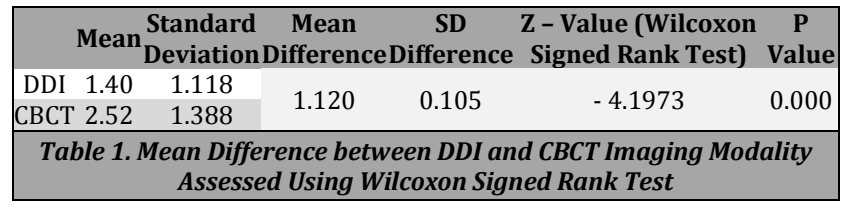

\section{DISCUSSION}

CBCT imaging which is three dimensional has more advantages than DDI and is useful to a greater extent in endodontics. The major disadvantage of DDI is the superimposition of surrounding structures and that's because of compressing a 3D object into a $2 \mathrm{D}$ image. ${ }^{22}$ CBCT with lesser FOV has no superimpositions of adjacent structures, higher signal to noise ratio and higher image contrast. ${ }^{23}$ Before planning an apical surgery a thorough and sound knowledge of surrounding vital structures such as maxillary sinus, mandibular canal, divergence and location of roots in close proximity of structures and identification of perforation of cortical bone and extension of the lesion into the maxillary sinus are essential. ${ }^{24}$

A study conducted by Francisco et al. reported a higher incidence of periapical lesion in 40 to 50 (66\%) years while in our study the highest prevalence was in 31 to 40 years (40 $\%) 30$ males (64\%) were affected predominantly and the results were similar to the study conducted by Arpita Rai. ${ }^{25}$ Periapical lesions were more frequently reported in maxillary anterior tooth regions; similar results have been reported by AO Akinyamoju. ${ }^{26}$

A total of 5 lesions that were undetected on DDI were detected on CBCT and scores of 4 and 5 were recorded more with CBCT images. Wilcoxon signed rank test showed that the values generated by the two methods differed statistically. This indicates that these lesions are better identified with a CBCT scan. Similar studies conducted by Arpita Rai et al. and Sara Lofthag - Hansen et al. reported comparable results.

\section{CONCLUSIONS}

Cone beam computed tomography with mid FOV has distinct advantages over DDI in detection and estimation of the size, extent, surrounding structures and all other associated features of the periapical lesion as they can be viewed in all 3 planes with higher image quality. Early and precise diagnosis is a key factor responsible for the success of any treatment. Finally, this study emphasises the importance of CBCT in detecting periapical lesions that are often overlooked or misdiagnosed on DDI. CBCT scans are higher in cost and radiation but the diagnostic accuracy is way higher, thus recommended as the diagnostic modality in the detection of periapical lesions.

\section{Limitations}

They are the cost, higher radiation exposure, lesser availability of the machine, artifacts and professional training in interpreting the images. The new concept of as low as diagnostically acceptable (ALADA) can be used in the optimization of radiographic exposure as sufficient for diagnosis. Collimating the beam, raising the chin, and protecting with thyroid collars and cervical spine shielding can all help to reduce radiation dose.

Data sharing statement provided by the authors is available with the full text of this article at jemds.com.

Financial or other competing interests: None.

Disclosure forms provided by the authors are available with the full text of this article at jemds.com.

\section{REFERENCES}

[1] Karjodkar FR. Infections and inflammatory lesions and systemic diseases affecting the jaws. In: Essentials of oral and maxillofacial radiology. Jaypee Brothers Medical Publishers 2014: p. 273.

[2] Gbadebo SO, Akinyamoju AO, Sulaiman AO. Periapical pathology: comparison of clinical diagnosis and histopathological findings. J West Afr Coll Surg 2014;4(3):74-88.

[3] Kamble AP, Pawar RR, Mattigatti S, et al. Cone-beam computed tomography as advanced diagnostic aid in endodontic treatment of molars with multiple canals: Two case reports. J Conserv Dent 2017;20(4):273-7.

[4] Juerchott A, Pfefferle T, Flechtenmacher C, et al. Differentiation of periapical granulomas and cysts by using dental MRI: a pilot study. Int J Oral Sci 2018;10(2):17.

[5] Grondahl HG, Huumonen S. Radiographic manifestations of periapical inflammatory lesions. How new radiological techniques may improve endodontic diagnosis and treatment planning. Endodontic Topics 2004;8(1):5567.

[6] Sogur E, Baksi BG, Gröndahl HG, et al. Detectability of chemically induced periapical lesions by limited cone beam computed tomography, intra-oral digital and conventional film radiography. Dentomaxillofac Radiol 2009;38(7):458-64.

[7] Schmidt JC, Gutekunst CJ, Dagassan-Berndt D, et al. Comparison of two-dimensional and three-dimensional radiographs using clinically relevant parameters. Dent J 2019;7(2):50.

[8] Kundel HL, Revesz G. Lesion conspicuity, structured noise, and film reader error. AJR Am J Roentgenol 1976;126(6):1233-8.

[9] Schwartz SF, Foster JK. Roentgenographic interpretation of experimentally produced bony lesions. Part I. Oral Surg Oral Med Oral Pathol 1971;32(4):606-12.

[10] Wallace JA, Nair MK, Colaco MF, et al. A comparative evaluation of the diagnostic efficacy of film and digital sensors for detection of simulated periapical lesions. Oral Surg Oral Med Oral Pathol Oral Radiol Endod 2001;92(1):93-7.

[11] Eickholz P, Hausmann E. Accuracy of radiographic assessment of interproximal bone loss in intrabony defects using linear measurements. Eur J Oral Sci 2000;108:70-73.

[12] Diwakar NR, Kamakshi SS. Recent advancements in dental digital radiography. Journal of Medicine, Radiology, Pathology and Surgery 2015;1:11-16.

[13] Ravi V, Lipee P, Rao CVN, et al. Direct digital radiography 
versus conventional radiography-assessment of visibility of file length placed in the root canal: an in vitro study. J Pharm Bioallied Sci 2012;4(Suppl 2):S285-9.

[14] Mao T, Neelakantan P. Three-dimensional imaging modalities in endodontics. Imaging Sci Dent 2014;44(3):177-83.

[15] Al Najjar A, Colosi D, Dauer LT, et al. Comparison of adult and child radiation equivalent doses from 2 dental cone beam computed tomography units. Am J Orthod Dentofacial Orthop 2013;143(6):784-92.

[16] Pauwels R, Araki K, Siewerdsen JH, et al. Technical aspects of dental CBCT: state of the art. Dentomaxillofac Radiol 2015;44(1):20140224.

[17] Iwai K, Arai Y, Hashimoto K, et al. Estimation of effective dose from limited cone beam X - ray CT examination. Shika Hoshasen 2000;40(4):251-9.

[18] Jaju PP, Jaju SP. Clinical utility of dental cone-beam computed tomography: current perspectives. Clin Cosmet Investig Dent 2014;6:29-43.

[19] Shenoy N, Ahmed J, Mallya SM. Add a third dimension to your patient care with cone beam computed tomography. J Interdiscip Dent 2014;4:118.

[20] Scarfe WC, Levin MD, Gane D, et al. Use of cone beam computed tomography in endodontics. Int J Dent 2009;2009:634567.
[21] Estrela C, Bueno MR, Azevedo BC, et al. A new periapical index based on cone beam computed tomography. J Endod 2008;34(11):1325-31.

[22] Venkatesh E, Elluru SV. Cone beam computed tomography: basics and applications in dentistry. J Istanb Univ Fac Dent 2017;51(3 Suppl 1):S102-21.

[23] Shah N, Bansal N, Logani A. Recent advances in imaging technologies in dentistry. World J Radiol 2014;6(10):794-807.

[24] Cotton TP, Geisler TM, Holden DT, et al. Endodontic applications of cone - beam volumetric tomography. J Endod 2007;33(9):1121-32.

[25] Rai A, Burde K, Guttal K, et al. Comparison between cone - beam computed tomography and direct digital intraoral imaging for the diagnosis of periapical pathology. J Oral Maxillofac Radiol 2016;4(3):50-6.

[26] Akinyamoju AO, Gbadebo SO, Adeyemi BF. Periapical lesions of the jaws: a review of 104 cases in ibadan. Ann Ib Postgrad Med 2014;12(2):115-9.

[27] Lofthag-Hansen S, Huumonen S, Gröndahl K, et al. Limited cone-beam CT and intraoral radiography for the diagnosis of periapical pathology. Oral Surg Oral Med Oral Pathol Oral Radiol Endod 2007;103(1):114-9. 